\title{
Effects of normal viscous stresses on radial viscous fingering
}

\author{
Hermes Gadêlha* \\ Centre for Mathematical Biology, Mathematical Institute, University of Oxford, 24-29 St. Giles, Oxford OX1 3LB, United Kingdom \\ and The Capes Foundation, Ministry of Education of Brazil, Caixa Postal 365, Brasília 70359-970, DF, Brazil
}

\author{
José A. Miranda ${ }^{\dagger}$ \\ Departamento de Física, LFTC, Universidade Federal de Pernambuco, Recife, Pernambuco 50670-901, PE, Brazil
}

(Received 6 April 2009; published 19 June 2009)

\begin{abstract}
We revisit the radial viscous fingering problem in a Hele-Shaw cell, and consider the action of viscous stresses originated from velocity gradients normal to the fluid-fluid interface. The evolution of the interface during linear and weakly nonlinear stages is described analytically through a mode-coupling approach. We find that the introduction of normal stresses influences the stability and the ultimate morphology of the emerging patterns. Although at early stages normal stresses tend to stabilize the interface, they act to favor the development of tip-splitting phenomena at the weakly nonlinear regime. We have also verified that finger competition events are only significantly affected by normal stresses for circumstances involving the development of a large number of interfacial fingers.
\end{abstract}

DOI: 10.1103/PhysRevE.79.066312

PACS number(s): 47.15.gp, 47.54.-r, 47.20.Ma, 47.15.km

\section{INTRODUCTION}

Since the seminal works by Bataille [1], Wilson [2], and Paterson [3] the radial viscous fingering problem has been extensively studied [4], both experimentally and theoretically. It constitutes a variation of the celebrated SaffmanTaylor problem in rectangular channels [5]. Under radial flow circumstances a less viscous fluid is radially injected into the narrow gap of a Hele-Shaw cell, which was previously filled with a more viscous fluid. Initially, the fluid-fluid interface is circular, but as time progresses, the interface undulates and fingerlike structures grow. Eventually, fingers of different lengths compete, spread and start to split at their tips, creating fanlike, branched patterns [6-8]. These experimental findings have been accurately modeled by intensive numerical simulations $[9,10]$. Based on all these facts, it is well established that spreading, splitting and competition are the three basic growth mechanisms of the radial viscous fingering process.

Despite all the progress made in the understanding of the radial fingering problem, the influence of normal viscous stresses on the dynamical evolution of the patterns, and their effects on the ultimate shape of the interface have been largely neglected. These stresses originated from velocity gradients acting normal to the interface [11]. Although normal stresses have no role in the rectangular version of the Hele-Shaw problem [12], it has been shown that they may have a significant impact in radial flow situations. Indeed, a few recent studies have demonstrated that normal viscous stresses act as an important element in determining the shape of rising patterns in the centrifugally driven rotating HeleShaw problem $[12,13]$, and also on the flow of magnetic and nonmagnetic fluids in variable-gap Hele-Shaw cells [14]. However, to our surprise, except for the initial efforts briefly

\footnotetext{
*gadelha@maths.ox.ac.uk

†jme@df.ufpe.br
}

presented by Joseph and collaborators [15], the action of normal viscous stresses on the traditional radial viscous fingering problem has been widely overlooked. A more thorough study about it is lacking.

In contrast to the vast majority of works in radial HeleShaw flows, we investigate how normal viscous stresses influence the interfacial evolution during linear and weakly nonlinear stages. The impact of such stresses is accounted for through a modified Young-Laplace pressure jump interfacial boundary condition. Within this context, we study how the stability properties of the interface, and the shape of the emerging patterns respond to the action of these stresses. At early linear stages it is found that normal stresses stabilize the system, in the sense that they reduce the number of resulting fingers. This characterizes a sort of effective surfacetension behavior. However, at the onset of nonlinear stages, we have verified that normal stresses do favor the occurrence of finger tip-splitting. Besides, finger competition can be restrained by normal stresses for large interfacial wave numbers. These facts indicate that normal viscous stresses are of relevance to a proper description of the interface behavior, and therefore should not be neglected.

\section{GOVERNING EQUATIONS}

Consider a Hele-Shaw cell of gap spacing $b$ containing two immiscible, incompressible, viscous fluids (see Fig. 1). Denote the viscosities of the inner and outer fluids, respec-

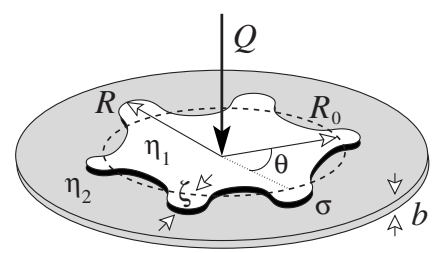

FIG. 1. Schematic representation of the radial viscous fingering problem in a Hele-Shaw cell. 
tively as $\eta_{1}$ and $\eta_{2}$. Between the two fluids there exists a surface tension $\sigma$. Fluid 1 is injected into fluid 2 at constant flow rate $Q$, equal to the area covered per unit time.

We describe the perturbed fluid-fluid interface as $\mathcal{R}(\theta, t)$ $=R(t)+\zeta(\theta, t)$, where $\theta$ represents the polar angle, $R(t)$ is the time dependent unperturbed radius

$$
R(t)=\sqrt{R_{0}^{2}+\frac{Q t}{\pi}},
$$

with $R_{0}$ being the unperturbed radius at $t=0$. In addition,

$$
\zeta(\theta, t)=\sum_{n=-\infty}^{+\infty} \zeta_{n}(t) \exp (\text { in } \theta)
$$

denotes the net interface perturbation with Fourier amplitudes $\zeta_{n}(t)$, and discrete azimuthal wave numbers $n$ $=0, \pm 1, \pm 2, \ldots$. Our perturbative approach keeps terms up to the second order in $\zeta$. In the Fourier expansion [Eq. (2)] we include the $n=0$ mode to maintain the area of the perturbed shape independent of the perturbation $\zeta$. Mass conservation imposes that the zeroth mode is written in terms of the other modes as $\zeta_{0}=-(1 / 2 R) \underset{n \neq 0}{\sum}\left|\zeta_{n}(t)\right|^{2}$.

For the quasi-two-dimensional geometry of the HeleShaw cell, the flow velocity $\mathbf{v}_{j}=-\nabla \phi_{j}$, where $\phi_{j}$ represents the velocity potential in fluids $j=1,2$. The equation of motion of the interface is given by Darcy's law $[5,16]$

$$
A\left(\frac{\phi_{2}+\phi_{1}}{2}\right)+\left(\frac{\phi_{2}-\phi_{1}}{2}\right)=-\frac{b^{2}\left(p_{1}-p_{2}\right)}{12\left(\eta_{1}+\eta_{2}\right)},
$$

where the dimensionless parameter $A=\left(\eta_{2}-\eta_{1}\right) /\left(\eta_{2}+\eta_{1}\right)$ is the viscosity contrast, and $p_{j}$ represents the hydrodynamic pressure.

To include the contributions coming from surface tension and viscous stresses we consider a generalized YoungLaplace pressure boundary condition, which expresses the jump in the normal stress across the fluid-fluid interface [12-14]

$$
\mathbf{n} \cdot\left[\pi^{(2)}-\pi^{(1)}\right] \cdot \mathbf{n}=\sigma \kappa .
$$

The left-hand side expresses the normal stress difference between the fluids which balances the stress of interfacial tension. The term at the right-hand side of Eq. (4) represents the contribution related to surface tension and interfacial curvature $\kappa$, with $\mathbf{n}=\boldsymbol{\nabla}[r-\mathcal{R}(\theta, t)] /|\boldsymbol{\nabla}[r-\mathcal{R}(\theta, t)]|$ denoting the unit normal vector at the interface.

The stress tensor [11]

$$
\pi_{i k}=-p \delta_{i k}+\eta\left[\frac{\partial v_{i}}{\partial x_{k}}+\frac{\partial v_{k}}{\partial x_{i}}\right]
$$

includes a viscous friction term proportional to the fluids' viscosity $\eta, \delta_{i k}$ denotes the Kronecker delta function, and $v_{i}$ represents the $i$ th component of the fluids' velocity vector. We rewrite Eq. (5) for each fluid using polar coordinates, substitute the resulting expressions into Eq. (4), and evaluate it at the interface to get

$$
p_{1}-p_{2}=\sigma \kappa-2 \delta\left[\eta_{1} \frac{\partial^{2} \phi_{1}}{\partial r^{2}}-\eta_{2} \frac{\partial^{2} \phi_{2}}{\partial r^{2}}\right]
$$

Note that the second term at the right-hand side of Eq. (6) takes into account stresses originated from normal velocity gradients. The parameter $\delta[\delta=1(\delta=0)$ if normal stresses are (not) considered] is used to keep track of the contributions coming from the normal stress term in Eq. (6).

The problem is then specified by the generalized pressure jump boundary condition Eq. (6), plus the kinematic boundary condition, which states that the normal components of each fluid's velocity are continuous at the interface

$$
\mathbf{n} \cdot \nabla \phi_{1}=\mathbf{n} \cdot \nabla \phi_{2} .
$$

We adapt a weakly nonlinear approach originally developed to study radial flow in Hele-Shaw cells without including normal stresses [16]. First, we define Fourier expansions for the velocity potentials, which obey Laplace's equation $\boldsymbol{\nabla}^{2} \phi_{j}=0$. Then, we express $\phi_{j}$ in terms of the perturbation amplitudes $\zeta_{n}$ by considering the kinematic boundary condition (7). Substituting these relations, and the modified pressure jump condition Eq. (6) into Eq. (3), always keeping terms up to second order in $\zeta$, and Fourier transforming, we find the dimensionless equation of motion for the perturbation amplitudes (for $n \neq 0$ )

$$
\dot{\zeta}_{n}=\lambda(n) \zeta_{n}+\sum_{n^{\prime} \neq 0}\left[F\left(n, n^{\prime}\right) \zeta_{n^{\prime}} \zeta_{n-n^{\prime}}+G\left(n, n^{\prime}\right) \dot{\zeta}_{n^{\prime}} \zeta_{n-n^{\prime}}\right]
$$

where the overdot denotes total time derivative, and

$$
\lambda(n)=\frac{1}{J(n)}\left\{\frac{1}{R^{2}}\left[A|n|\left(1+\delta \frac{b^{2}}{3 R^{2}}\right)-J(n)\right]-\frac{b^{2} B}{6 R^{3}}|n|\left(n^{2}-1\right)\right\}
$$

is the linear growth rate, with

$$
J(n)=\left[1+\delta \frac{b^{2}}{6 R^{2}}|n|(|n|+A)\right] .
$$

The dimensionless parameter

$$
B=\frac{\sigma \pi R_{0}}{|Q|\left(\eta_{1}+\eta_{2}\right)}
$$

measures the relative strength between surface tension and viscous forces.

The second-order mode-coupling terms are given by

$$
\begin{aligned}
F\left(n, n^{\prime}\right)= & \frac{|n|}{J(n)}\left\{\frac{\operatorname{sgn}(Q)}{R^{3}}\left[A\left(\frac{1}{2}-\operatorname{sgn}\left(n n^{\prime}\right)\right)+\delta \frac{b^{2}}{6 R^{2}} f_{1}\left(n, n^{\prime}\right)\right]\right. \\
& \left.-\frac{b^{2} B}{6 R^{4}}\left[1-\frac{n^{\prime}}{2}\left(3 n^{\prime}+n\right)\right]\right\},
\end{aligned}
$$

and 


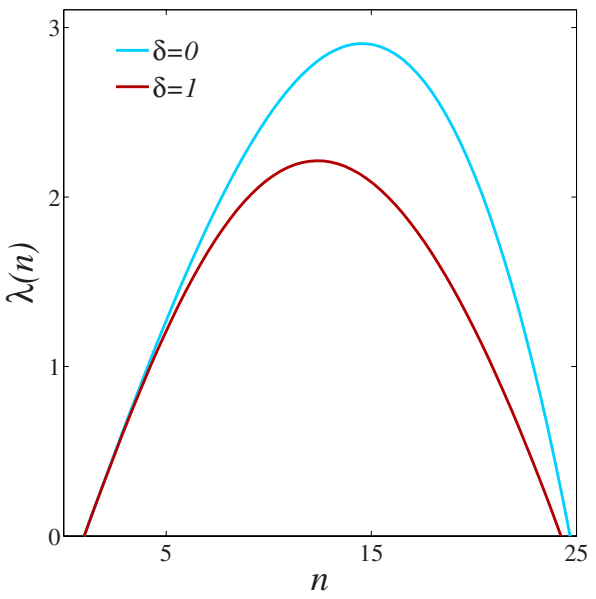

FIG. 2. (Color online) Linear growth rate $\lambda(n)$ as a function of mode $n$, for $A=1, B=0.64, b=0.16$, and $\delta=0,1$, at time $t=1$.

$G\left(n, n^{\prime}\right)=\frac{1}{J(n)}\left\{\frac{|n|}{R}\left[A\left[1-\operatorname{sgn}\left(n n^{\prime}\right)\right]+\delta \frac{b^{2}}{6 R^{2}} f_{2}\left(n, n^{\prime}\right)\right]-\frac{1}{R}\right\}$

where

$$
\begin{aligned}
f_{k}\left(n, n^{\prime}\right)= & \left\{A\left[(-1)^{k}+n^{\prime 2}-n^{2} \operatorname{sgn}\left(n n^{\prime}\right)\right]\right. \\
& \left.-|n|\left[k-1+\operatorname{sgn}\left(n n^{\prime}\right)\right]+3\left|n^{\prime}\right|\right\},
\end{aligned}
$$

and $k=1,2$.

The sgn function equals \pm 1 according to the sign of its argument. In Eq. (8) lengths are rescaled by $R_{0}$, and velocities by $|Q| /\left(2 \pi R_{0}\right)$. From now on, we work with the dimensionless version of the equations. After appropriate reintroduction of dimensions, it can be shown that the functions $\lambda(n), F\left(n, n^{\prime}\right)$, and $G\left(n, n^{\prime}\right)$ reduce to the equivalent expressions obtained in Ref. [16] in the zero normal stress limit $(\delta=0)$. Although $Q$ can be either positive (injection) or negative (suction), we focus on the traditional case $Q>0$. Moreover, we consider the unstable regime in which $A \geq 0$. We stress that the values we take for our dimensionless parameters are consistent with typical physical quantities used in real experiments in radial viscous flow $[1,3,4,6-8]$.

\section{LINEAR AND WEAKLY NONLINEAR REGIMES}

In this section we investigate the consequences of the changes introduced by the inclusion of normal viscous stresses in both linear and weakly nonlinear stages of the interface evolution. We focus on two basic questions: (i) at the linear level, what are the changes on the linear growth rate? (ii) regarding the onset of nonlinear effects, how do finger tip-splitting and finger competition are influenced by normal stresses?

We begin by using the linear growth rate [Eq. (9)] to gain insight about the influence of the normal stresses at the linear stage of pattern evolution. Figure 2 plots $\lambda(n)$ as a function of mode number $n$ for $A=1, B=0.64, b=0.16, t=1$, and $\delta$ $=0,1$. The most evident feature in Fig. 2 is the fact that the inclusion of viscous stresses $(\delta=1)$ leads to a decreased growth rate of the fastest growing mode $n_{\max }$ [obtained by setting $d \lambda(n) / d n=0$ ], shifting it toward lower values of azimuthal wave numbers. In Fig. $2 n_{\max }$ changes from approximately 14 to 12 due to normal stresses. We have verified that this shift is increasingly larger for smaller values of $B$. Since $n_{\max }$ determines the typical number of fingers formed at a given time, this means that by considering the normal stresses one should expect slower growth, and possibly patterns with a smaller number of fingers. In this sense, these stresses act to stabilize the interface assuming the role of an effective surface tension. On the other hand, we notice that the band of unstable modes [estimated by setting $\lambda(n)=0$ ] remains nearly unchanged. These linear stability results are consonant with the similar linear findings presented in Refs. [12-14].

An illustrative purely linear evolution of the interface is depicted in Fig. 3 which considers the participation of two generic cosine modes [a fundamental $n=6$ and its harmonic $2 n=12]$. The evolving interfaces are taken at equal time intervals, for: (a) $\delta=0$, and (b) $\delta=1$. In (c) the final time interfaces [taken at $t=4.58$ ] obtained in (a) and (b) are plotted together to facilitate comparison. We set $A=1, B=0.64$, and $b=0.16$. The initial amplitudes are $a_{n}(0)=3.1 \times 10^{-3}$, and $a_{2 n}(0)=10^{-6}\left[\right.$ note that $\left.a_{2 n}(0) \ll a_{n}(0)\right]$. We stop the time evolution of the patterns shown in Fig. 3 as soon as the base of the fingers starts to move inward, what would make successive interfaces to cross one another. Since this crossing is not observed in experiments [1,3,4,6-8], we follow Ref. [17] and adopt the largest time before crossing as the upper bound time for the validity of our theoretical description. Regardless the value of $\delta$ the resulting patterns are sixfold symmetric, presenting the formation of fingering structures which show no tendency toward finger broadening and finger tip splitting. However, in the presence of normal stresses pattern evolution is delayed, so that the lengths of the outward moving fingers are smaller, illustrating the stabilizing nature and the surface-tension-like role of viscous stresses at purely linear stages.

One must go beyond purely linear analysis in order to systematically investigate the main morphological features possibly induced by normal stresses. To do that we now turn our attention to the weakly nonlinear flow stage, and begin by discussing finger tip-splitting events. Despite the somewhat complicated nature of the mode-coupling Eq. (8), we will see that valuable information can be extracted from it by examining the coupling of a small number of Fourier modes. To simplify our discussion we rewrite Eq. (8) in terms of cosine and sine modes, where the cosine $a_{n}=\zeta_{n}+\zeta_{-n}$ and sine $b_{n}=i\left(\zeta_{n}-\zeta_{-n}\right)$ amplitudes are real valued. For consistent second-order expressions, we replace the time derivative terms $\dot{a}_{n}$ and $\dot{b}_{n}$ by $\lambda(n) a_{n}$ and $\lambda(n) b_{n}$, respectively. Without loss of generality we choose the phase of the fundamental mode so that $a_{n}>0$ and $b_{n}=0$. Under such circumstances, finger tip-splitting phenomena are described by considering the influence of a fundamental mode $n$ on the growth of its harmonic $2 n$ [16]. The equations of motion for the harmonic mode are quite simple, and can be written as

$$
\dot{a}_{2 n}=\lambda(2 n) a_{2 n}+T(2 n, n) a_{n}^{2},
$$



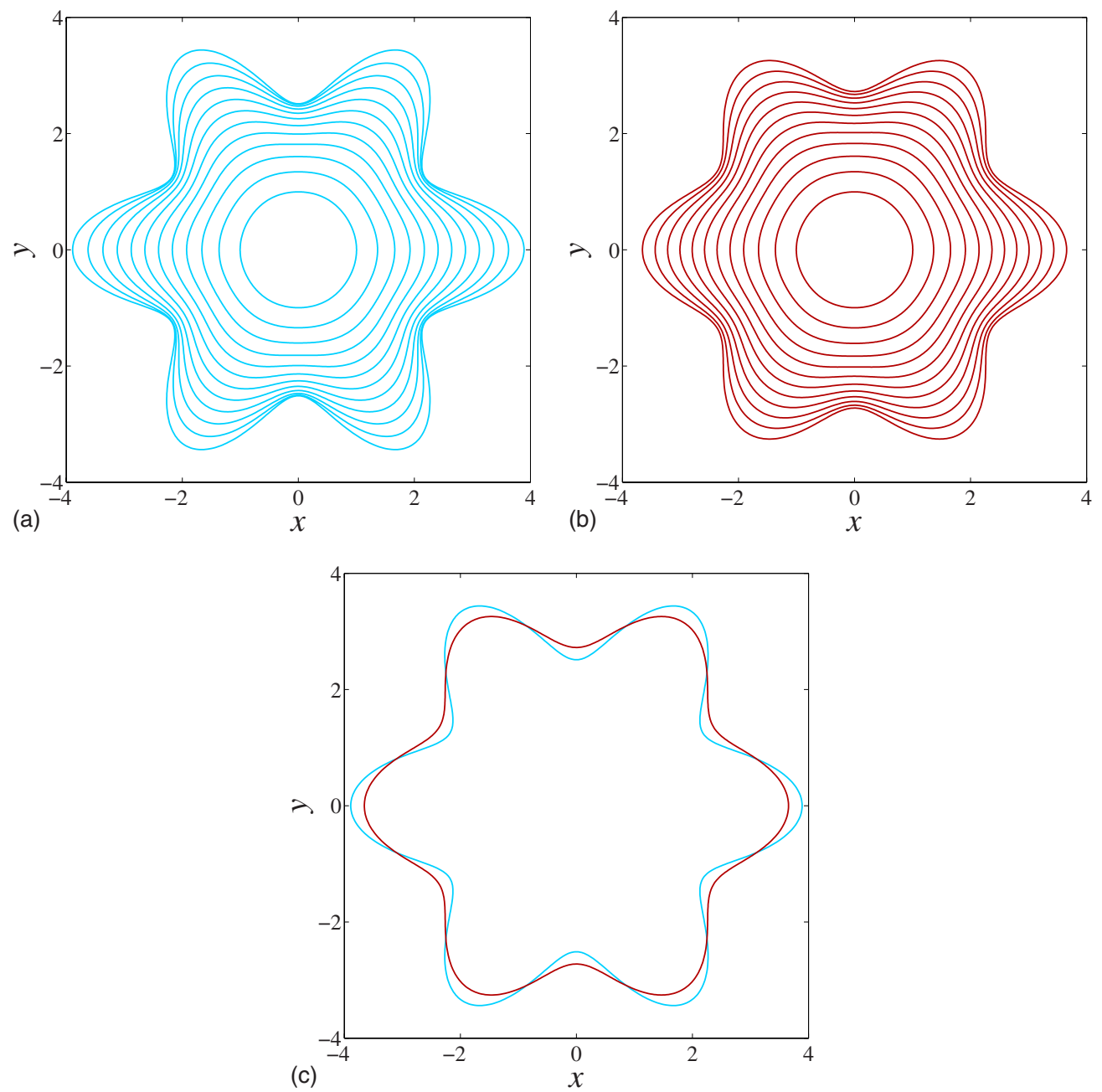

FIG. 3. (Color online) Snapshots of the purely linear evolution of the interface, plotted at equal time intervals including two representative cosine modes $n=6$ and $2 n=12$ when (a) $\delta=0$, and (b) $\delta=1$. The final time interfaces plotted in (a) and (b) are overlaid in (c). Note that the radial growth of the fingers moving outward tends to be suppressed when $\delta=1$.

$$
\dot{b}_{2 n}=\lambda(2 n) b_{2 n},
$$

where the finger tip function is defined as

$$
T(2 n, n)=\frac{1}{2}[F(2 n, n)+\lambda(n) G(2 n, n)] .
$$

Since the growth of the sine mode $b_{2 n}$ is uninfluenced by $a_{n}$ and does not present second-order couplings, we focus on the growth of the cosine mode $a_{2 n}$ as given by Eq. (15). It shows that the presence of the fundamental mode $n$ forces growth of the harmonic mode $2 n$. The function $T(2 n, n)$ acts like a driving force and its sign dictates if finger tip-splitting is favored or not by the dynamics. If $T(2 n, n)<0, a_{2 n}$ is driven negative, precisely the sign that leads to finger tip broadening and finger tip splitting. If $T(2 n, n)>0$ growth of $a_{2 n}>0$ would be favored, leading to outward-pointing finger tip narrowing.

It is important to stress that the driving term in Eq. (15) spontaneously induces the growth of the harmonic mode. Therefore, from our second-order mode-coupling approach finger broadening and finger tip splitting are obtained even in the absence of random noise, and without artificially introducing the presence of a sizable harmonic through initial conditions. On the other hand, these maneuvers or the consideration of unrealistic long times would be necessary to observe finger broadening and tip-splitting events by using a purely linear theory. In this sense, these two growth mechanisms are unique predictions of an intrinsically nonlinear theory.

To analyze the influence of normal viscous stresses on the tip-splitting behavior at second order, in Fig. 4 we plot the behavior of $T(2 n, n)$ as a function of the viscosity contrast $A$, for two Fourier modes $(n=4$ and $n=6)$. This is done for the situations in which normal viscous stresses are neglected $(\delta=0)$, and taken into consideration $(\delta=1)$. We set $B=0.64$, $b=0.16$, and $t=5.56$. Except for a few smaller values of $A$ for which $T(2 n, n) \geq 0$, we see that $T(2 n, n)$ is mostly negative, indicating tendency toward finger tip splitting. Most interestingly, we can also observe that, regardless the value of $n$, the curves associated with $\delta=1$ lie below the corresponding curves for $\delta=0$. This indicates that the fingers have an enhanced tendency to split at their tips, due to the effects of normal viscous stresses. 


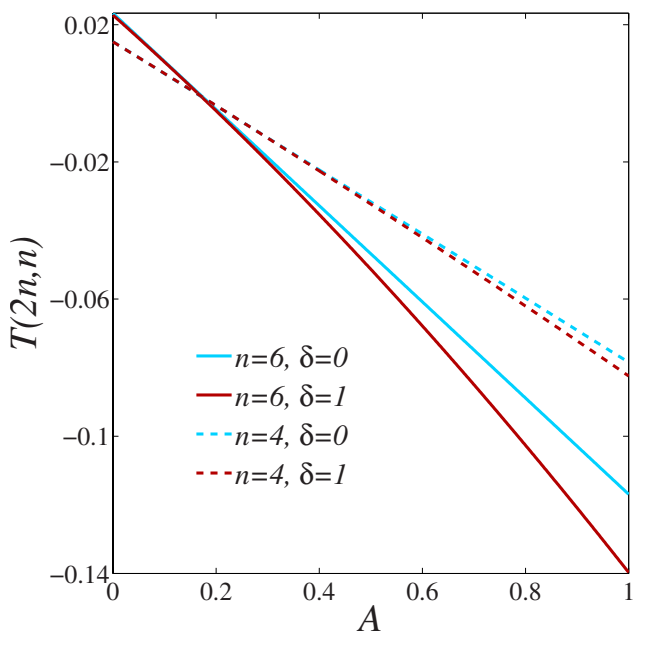

FIG. 4. (Color online) Behavior of the function $T(2 n, n)$ in terms of the viscosity contrast $(0 \leq A \leq 1)$, for two values of mode $n$, both in the presence $(\delta=1)$, and absence $(\delta=0)$ of normal viscous stresses.

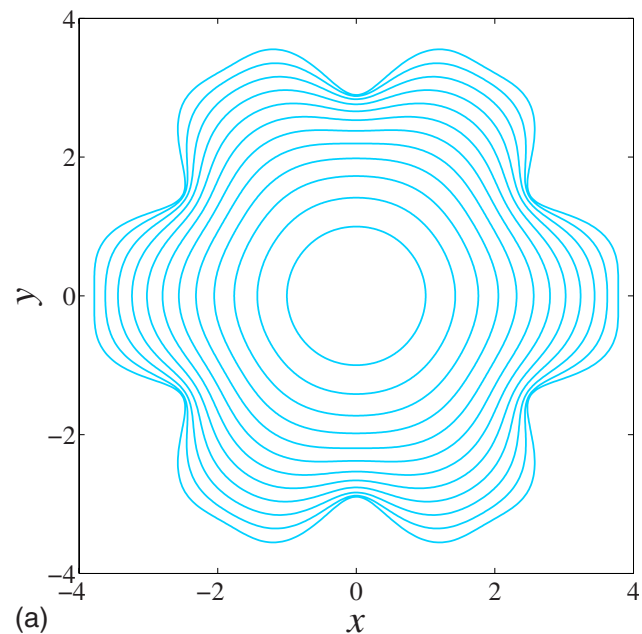

The role of normal viscous stresses in determining the finger tip-splitting behavior is even more clearly illustrated in Fig. 5 which plots the time evolution of the interface, plotted at equal time intervals, considering the interaction of two representative cosine modes [a fundamental $n=6$ and its harmonic $2 n=12$ ], for: (a) $\delta=0$, and (b) $\delta=1$. In (c) the final time interfaces [taken at $t=5.62$ ] obtained in (a) and (b) are plotted together. Again, we set $A=1, B=0.64$, and $b=0.16$. The initial amplitudes are $a_{n}(0)=1.39 \times 10^{-3}$, and $a_{2 n}(0)$ $=10^{-5}$ [note that $\left.a_{2 n}(0) \ll a_{n}(0)\right]$. Note that differently from the purely linear picture of the phenomenon, in Fig. 5 the modes $n=6$ and $n=12$ are not independent, but coupled to each other.

In Fig. 5(a) we see a nearly circular initial interface evolving to a six-fingered structure. Finger broadening can be observed and, at later times, the finger tips become increasingly flat. However, the finger tips do not bifurcate. It is also worth noting that a similar, but purely linear description of the interface motion as illustrated by Fig. 3(a) leads to patterns presenting fingers that are sharper than the fingers obtained Fig. 5(a). The development of broad fingers in Fig. 5(a) results from nonlinear effects.

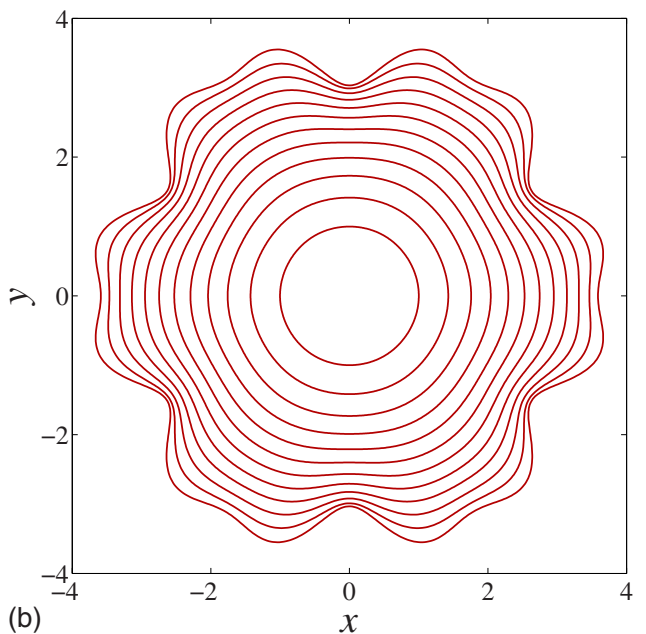

(b)

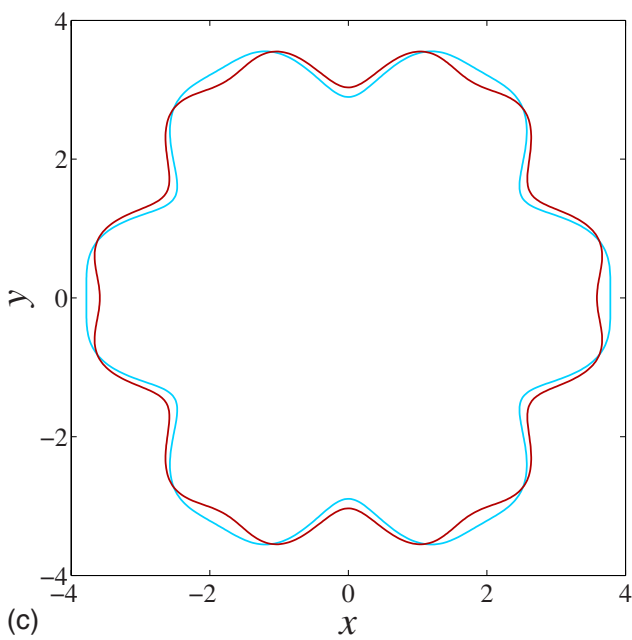

FIG. 5. (Color online) Snapshots of the evolving the interface, plotted at equal time intervals for the interaction of two cosine modes $n=6$ and $2 n=12$ when (a) $\delta=0$, and (b) $\delta=1$. The final time interfaces plotted in (a) and (b) are overlaid in (c). Finger tip-splitting is favored when $\delta=1$. 
The scenario presented in Fig. 5(a) is changed when we include the action of normal viscous stresses. As one can see by examining Fig. 5(b) the presence of normal stresses leads to wider fingers, having blunt tips. At later times, these grown fingers clearly start to bifurcate, by splitting at their tips. The morphological differences between the final patterns shown in Figs. 5(a) and 5(b) are depicted in Fig. 5(c). On this basis, we can say that a stronger tendency toward splitting is observed when normal viscous stresses are taken into account. This indicates that such stresses play an important role in the occurrence of tip-splitting phenomena in radial viscous fingering.

The increased tendency toward finger tip-splitting illustrated in Fig. 5 can be interpreted by the effective surface tension role played by the normal viscous stresses, as commented during the discussion of the linear effects. By adding viscous stresses into the problem we are in fact increasing the net surface tension acting on the fingers. It is well known that surface tension is the physical force causing spreading and splitting [4]: a growing tip with surface tension does not stabilize, but undergoes a tip-splitting instability. This is because surface tension slows the growth of sharply curved surfaces. Therefore, it seems reasonable to expect that the presence of normal viscous stresses would lead to an enhanced tip-splitting behavior. In this sense viscous stresses play a dual role; linearly, they act to stabilize the system by decreasing the radial growth and the typical number of emerging fingers; but at the slightly nonlinear level they destabilize the fingers to splitting, ensuing further tip bifurcation.

At this point, we turn to the investigation of the influence of normal viscous stresses on the finger competition behavior. Considering the length variability as a measure of the finger competition, it can be described by the influence of a fundamental mode $n$, assuming $n$ is even, on the growth of its subharmonic mode $n / 2[13,16]$. The correctness and accuracy of this simple finger competition mechanism has been tested by sophisticated numerical simulations $[18,19]$. Within this approach and considering the same assumptions used to derive Eqs. (15) and (16), the equations of motion for the subharmonic mode can be written as

$$
\begin{aligned}
& \dot{a}_{n / 2}=\left\{\lambda(n / 2)+C(n) a_{n}\right\} a_{n / 2}, \\
& \dot{b}_{n / 2}=\left\{\lambda(n / 2)-C(n) a_{n}\right\} b_{n / 2},
\end{aligned}
$$

where the finger competition function is given by

$$
C(n)=\frac{1}{2}\left[F\left(-\frac{n}{2}, \frac{n}{2}\right)+\lambda(n / 2) G\left(\frac{n}{2},-\frac{n}{2}\right)\right] .
$$

Observing Eqs. (18) and (19) we verify that $C(n)>0$ increases the growth of the cosine subharmonic $a_{n / 2}$, while inhibiting growth of its sine subharmonic $b_{n / 2}$. The result is an increased variability among the lengths of fingers of the less viscous fluid 1 penetrating into the more viscous fluid 2 . This effect describes finger competition. Sine modes $b_{n / 2}$ would vary the lengths of fingers of the more viscous fluid 2 penetrating into the less viscous fluid 1, but it is clear from Eq. (19) that their growth is suppressed. Reversing the sign

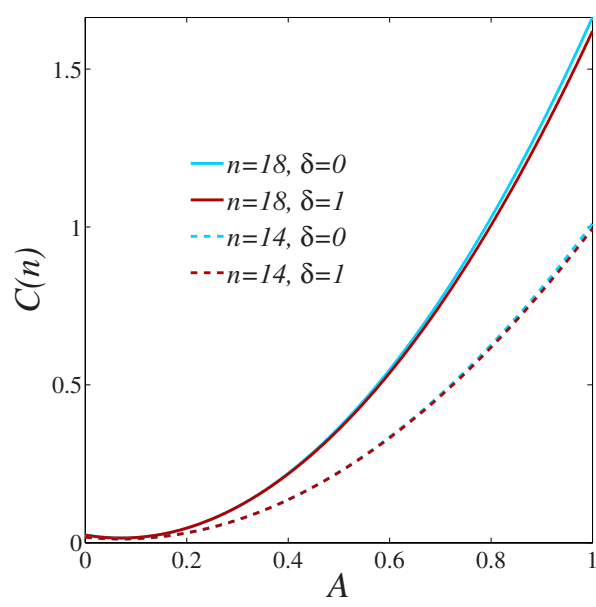

FIG. 6. (Color online) Finger competition function $C(n)$ as given by Eq. (20) in terms of the viscosity contrast $A(0 \leq A \leq 1)$, for two values of mode $n$, both in the presence $(\delta=1)$, and absence $(\delta=0)$ of normal viscous stresses.

of $C(n)$ would exactly reverse these conclusions, such that modes $b_{n / 2}$ would be favored over modes $a_{n / 2}$. Regardless of its sign, the function $C(n)$ measures the strength of the competition: increasingly larger values of $C(n)$ lead to enhanced finger competition.

Figure 6 illustrates how the finger competition function $C(n)$ varies with the viscosity contrast $A$ for two generic modes $n=14$ and $n=18$. We take $B=0.64, b=0.16$, and $t$ $=5.56$. Independently of the values of $n$ and $\delta$, we note that $C(n)>0$, indicating competition among the fingers moving outward. Moreover, it is evident that not much difference is obtained when normal viscous stresses are taken into consideration. The two $C(n)$ curves for mode $n=14$ are nearly indistinguishable, while the two corresponding curves for mode $n=16$ just present a small separation as $A \rightarrow 1$. Nevertheless, rigorously speaking the competition would be slightly decreased if normal stresses act, but again as we can see from Fig. 6 this would be a very mild effect. However, we also have verified that for larger modes (for instance, $n$ $=30$ or $n=40$ ) the decrease in finger competition due to normal stresses becomes more accentuated. This is an indication the finger competition would be more significantly changed by stresses for situations involving the development of a large number of fingers.

\section{CONCLUSIONS}

Although radial viscous fingering in Hele-Shaw cells is a vastly studied problem, the influence of normal viscous stresses on the stability and ultimate shape of the patterns has been markedly overlooked. In this work we tacked the problem by applying a mode-coupling approach. We performed calculations on a model which is simple enough to be amenable to analytical solution and yet retains the essential physics of the problem involving normal viscous stresses. As a result, linear contributions provide information about the instability of the interface, whereas weakly nonlinear terms allow us to access and predict important facts about the morphology of the patterns. 
Our linear results indicate that normal viscous stresses tend to stabilize the interface, slowing down its growth, and reducing the number of interfacial fingers. This attributes a surface tensionlike signature to normal stresses. At the weakly nonlinear regime, the stresses favor the occurrence of finger tip-splitting events, but act to diminish finger competition, mainly when the interface presents a large number of fingers. These findings indicate that normal viscous stresses can affect the basic growth mechanisms of radial viscous fingering, and therefore should not be neglected.

Our theoretical work makes specific predictions that have not yet been tested experimentally. One possible experimental check about the role of normal stresses could be performed by investigating the number fingers emerging at early states of pattern evolution. By focusing on the high viscosity contrast $A$ (e.g., $A \rightarrow 1)$ and low $B(B \sim 0.01-0.1)$ limits, one could compare the experimental data for the actual number of emerging fingers, with the fastest growing mode $\left(n_{\max }\right)$ predicted by our linear stability analysis, both in the absence $(\delta=0)$ and presence $(\delta=1)$ of the normal stress contribution. A similar experimental test has been performed in Ref. [12] where the influence of normal stresses on the morphology of patterns in rotating Hele-Shaw cells has been examined. Additional tests, now related to fully nonlinear stages of the dynamics, could also be performed by implementing extensive numerical simulations (for instance, by using boundary integral techniques $[9,18]$ ) that take into account our modified pressure boundary condition [Eq. (6)]. This would allow one to scrutinize systematically the predicted stress-induced enhancement of finger tip-splitting at later stages. In summary, we hope our current work will stimulate further investigations about the role of normal viscous stresses on the radial viscous fingering problem.

\section{ACKNOWLEDGMENTS}

J.A.M. thanks CNPq (Brazilian Research Council) for financial support of this research through the program "Instituto Nacional de Ciência e Tecnologia de Fluidos Complexos (INCT-FCx)," and also through the CNPq/FAPESQ Pronex program. H.G. wishes to thank CAPES (Brazilian sponsor) for financial support through Grant No. BEX 4676/06-8.
[1] J. Bataille, Rev. Inst. Fr. Pet. Ann. Combust. Liq. 23, 1349 (1968).

[2] S. D. R. Wilson, J. Colloid Interface Sci. 51, 532 (1975).

[3] L. Paterson, J. Fluid Mech. 113, 513 (1981).

[4] For review articles see for instance, G. Homsy, Annu. Rev. Fluid Mech. 19, 271 (1987); K. V. McCloud and J. V. Maher, Phys. Rep. 260, 139 (1995); J. Casademunt, Chaos 14, 809 (2004).

[5] P. G. Saffman and Geoffrey Taylor, Proc. R. Soc. London, Ser. A 245, 312 (1958).

[6] J.-D. Chen, J. Fluid Mech. 201, 223 (1989); Exp. Fluids 5, 363 (1987).

[7] H. Thomé, M. Rabaud, V. Hakim, and Y. Couder, Phys. Fluids A 1, 224 (1989).

[8] O. Praud and H. L. Swinney, Phys. Rev. E 72, 011406 (2005).

[9] P. Fast and M. J. Shelley, J. Comput. Phys. 212, 1 (2006).

[10] J. Mathiesen, I. Procaccia, H. L. Swinney, and M. Thrasher, EPL 76, 257 (2006).
[11] L. D. Landau and E. M. Lifshitz, Course of Theoretical Mechanics: Fluid Mechanics (Pergamon Press, New York, 1959), Vol. 6.

[12] E. Alvarez-Lacalle, J. Ortín, and J. Casademunt, Phys. Fluids 16, 908 (2004).

[13] H. Gadêlha and J. A. Miranda, Phys. Rev. E 70, 066308 (2004).

[14] R. M. Oliveira and J. A. Miranda, Phys. Rev. E 73, 036309 (2006).

[15] D. D. Joseph, H. Kim, and T. Funada, Bull. Am. Phys. Soc. 53 (15), 183 (2008).

[16] J. A. Miranda and M. Widom, Physica D 120, 315 (1998).

[17] M. J. P. Gingras and Z. Rácz, Phys. Rev. A 40, 5960 (1989).

[18] J. A. Miranda and E. Alvarez-Lacalle, Phys. Rev. E 72, 026306 (2005).

[19] C.-Y. Chen, C.-H. Chen, and J. A. Miranda, Phys. Rev. E 73, 046306 (2006). 